\title{
Erratum: Design and application of fuel injection drive circuit for engine test bench
}

\author{
Baoqing Deng1, Yuchuan Jia ${ }^{2}$, Zhang Bo ${ }^{3}$, Zhenyu Deng ${ }^{4}$, Zengfa Gao ${ }^{5}$ \\ $1,3,4,5$ Mechanical Engineering, Zhuhai College of Jilin University, Zhuhai, 519041, China \\ ${ }^{2}$ GAC Passenger Car, Ltd, Guangzhou, China \\ ${ }^{3}$ School of Mechanical and Aerospace Engineering, Jilin University, Changchun, 130012, China \\ ${ }^{1}$ Corresponding author \\ E-mail: ${ }^{1}$ b999mng@163.com, ${ }^{2} 2300976323 @ q q . c o m,{ }^{3}$ blptx9@sina.com, ${ }^{4} d x 7 i 59 @ 163 . c o m$, \\ 5gaec78@163.com
}

DOI https://doi.org/10.21595/vp.2020.21837

Check for updates

Copyright $(2020$ Baoqing Deng, et al. This is an open access article distributed under the Creative Commons Attribution License, which permits unrestricted use, distribution, and reproduction in any medium, provided the original work is properly cited.

\section{Publisher's note regarding paper}

Deng Baoqing, Jia Yuchuan, Bo Zhang, Deng Zhenyu, Gao Zengfa Design and application of fuel injection drive circuit for engine test bench. Vibroengineering PROCEDIA, Vol. 33, 2020, p. 118-123, https://doi.org/10.21595/vp.2020.21524.

\section{The description of the correction}

The institutional affiliations were typed incorrectly in the paper finally approved (after the acceptance) by the authors.

Incorrect institutional affiliations:

1,3,4, 5 Mechanical Engineering, Zhuhai College of Jinlin University, Zhuhai, 519041, China

${ }^{2}$ GAC Passenger Car, Ltd, Guangzhou, China

${ }^{3}$ School of Mechanical and Aerospace Engineering, Jilin Universityy, Changchun, 130012, China

Revised institutional affiliations:

1,3, 4, 5 Mechanical Engineering, Zhuhai College of Jilin University, Zhuhai, 519041, China

${ }^{2}$ GAC Passenger Car, Ltd, Guangzhou, China

${ }^{3}$ School of Mechanical and Aerospace Engineering, Jilin University, Changchun, 130012, China 